\title{
The Critical Role of AKT2 in Hepatic Steatosis Induced by PTEN Loss
}

\author{
Lina $\mathrm{He},{ }^{*}$ Xiaogang Hou, ${ }^{*}$ Gary Kanel, ${ }^{\dagger}$ Ni Zeng, ${ }^{*}$ \\ Vivian Galicia, ${ }^{*}$ Ying Wang, ${ }^{\ddagger}$ Jian Yang, ${ }^{\S}$ \\ Hong Wu, ${ }^{\ddagger}$ Morris J. Birnbaum, ${ }^{\text {"T }}$ \\ and Bangyan L. Stiles* \\ From the Departments of Molecular Pharmacology and \\ Pharmaceutical Sciences," University of Southern California, Los \\ Angeles, California; the Department of Pathology, ${ }^{\dagger}$ Keck School of \\ Medicine, University of Southern California, Los Angeles, \\ California; the Department of Molecular Pharmacology and \\ Toxicology, ${ }^{\ddagger}$ David Geffen School of Medicine, University of \\ California, Los Angeles, California; the Department of \\ Physiology, ${ }^{\S}$ University of South Alabama College of Medicine, \\ Mobile, Alabama; and the Departments of Medicine, Cell and \\ Developmental Biology, and Pharmacology, "Iniversity of \\ Pennsylvania, Philadelphia, Pennsylvania
}

Insulin signaling in the liver leads to accumulation of phosphatidylinositol $(3,4,5)$-trisphosphate $\left(\mathbf{P I P}_{3}\right)$. Deletion of the phosphatase Pten (phosphatase and tensin homologue deleted on chromosome 10) reduces PIP $_{3}$ levels and leads to fatty liver development. The purpose of this study was to investigate the mechanisms underlying lipogenesis that result from $\mathbf{P I P}_{3}$ accumulation using liver Pten-deletion mice. To explore the role of AKT2, the major liver AKT isoform in steatosis induced by deletion of Pten, we created mice lacking both Pten and Akt 2 in hepatocytes and compared the effect of deleting Akt2 and Pten in the double mutants to the Pten deletion mice alone. Hepatic lipid accumulation was significantly reduced in mice lacking both PTEN and AKT2, as compared with Pten mutant mice alone. This effect was due to the role of AKT2 in maintaining expression of genes involved in de novo lipogenesis. We showed that lipid accumulation in the double mutant hepatocytes was partially reversed by expression of constitutive active FOXO1, a transcription factor downstream of AKT not dependent on inhibition of atypical protein kinase $C$. In summary, this study delineated regulation of lipid metabolism by PI3K signaling pathway by showing that AKT mediates PIP $_{3}$ accumulation (mimicked by PTEN loss) induced lipid deposition in the liver and provided an important molecular mechanism for in- sulin-regulated hepatic lipogenesis. (AmJ Pathol 2010, 176:2302-2308; DOI: 10.2353/ajpath.2010.090931)

Lipid phosphatase and tensin homologue deleted on chromosome 10 (PTEN) functions to convert phosphotidylinositol-3,4,5-trisphosphate $\left(\mathrm{PIP}_{3}\right)$ to phosphatidylinositol bisphosphate. ${ }^{1}$ Thus, PTEN antagonizes the insulin-phosphoinositide 3-kindase (PI3K)/AKT pathway by reducing the levels of $\mathrm{PIP}_{3}$. In the liver, insulin activates $\mathrm{PI} 3 \mathrm{~K}$ signal to drive hepatic lipid accumulation. Mice lacking all PI3K activities are hypolipidemic and exhibit reduced expression of lipogenic genes in the liver. ${ }^{2}$ Previous studies reported a liver steatosis phenotype in mice when PTEN is lost in the hepatocytes. ${ }^{3,4}$ In hepatocyte-specific Pten deletion mice, liver becomes a lipogenic organ, and accumulates triglyceride (TG) even though there is systemic hypoglycemia. ${ }^{4}$ Both sterol regulatory element-binding proteins (SREBP-1c) and fatty acid synthase (FAS) are markedly elevated when PTEN is lost in the hepatocyte. ${ }^{3,4}$ This model, thus, is relevant to study how activation of the PI3K/AKT signaling pathway, specifically $\mathrm{PIP}_{3}$ accumulation, induces lipogenesis.

In this study, we considered the role of AKT2 in mediating the lipogenic effect of insulin/PI3K signaling. AKT2 is the major isoform of AKT that is expressed in the liver. AKT plays a pivotal role in hepatic insulin action. ${ }^{5}$ Adenovirus expression of constitutively activated AKT in vivo led to a similar fatty liver phenotype as loss of PTEN. ${ }^{6}$ We hypothesized that AKT2 may mediate the metabolic phenotypes observed in the livers of Pten mutant animals. We investigated this hypothesis by crossing the hepatocytespecific Pten mutant mice ${ }^{4}$ with the $A k t 2^{-1-}$ mice. ${ }^{7}$ Our results indicate that loss of AKT2 can effectively block the fatty deposition in the liver of the Pten mutant mice. Our

Supported by the National Institute of Diabetes and Disease of the Kidney (NIDDK) and University of Southern California Liver Disease Center. M.J.B. acknowledges funding from NIDDK (R01-DK56886).

Accepted for publication January 19, 2010

Supplemental material for this article can be found on http://ajp. amjpathol.org

Address reprint requests to Bangyan Stiles, Ph.D., Molecular Pharmacology and Pharmaceutical Sciences, University of Southern California, Los Angeles, CA 90089, E-mail: bstiles@usc.edu. 
study shows that AKT2 is critical for the expression of lipogenic genes. This effect is independent of the atypical protein kinase $\mathrm{C}(\mathrm{aPKC})$ and may be partially dependent on the forkhead transcriptional factor FOXO1.

\section{Materials and Methods}

\section{Animals}

The Pten ${ }^{\text {loxPlloxP }} ;$ Allb-Cre ${ }^{+}\left(C^{+} ; A^{+/+}\right.$, Pten null $)$mice ${ }^{4}$ were crossed with $A k t 2^{-1-}\left(C^{-} ; A^{-1-}\right.$, Akt2 null $)$ mice $^{7}$ to generate the double mutant Pten ${ }^{\text {loxP/loxP }}$; Akt2 ${ }^{-1-}$; Allb$\mathrm{Cre}^{+}\left(C^{+} ; A^{-1-}, D M\right)$. Male mice were used for experiments. Controls are male Pten ${ }^{\text {IoxP/loxP }}$; Alb-Cre ${ }^{-}$mice. Experiments are performed following protocols approved by the University of Southern California Institutional Animal Care and Use Committee.

Fasting blood glucose levels were determined using a Therasense glucometer after overnight fasting. Livers were perfused with cold PBS and collected in formalin for histology, optimal cutting temperature compound for frozen section and flash frozen for protein, RNA and lipid analysis. Tissue sections were stained with H\&E and Oil Red O.

\section{Cell Lines, Plasmids, and Transfections}

Pten mutant and control hepatocyte cell lines were established from the Pten mutant and control mice as described previously. ${ }^{8}$ Plasmid for wild-type FOXO1-WT and FOXO1-AAA mutants are obtained from Add-gene. Plasmids were transfected into the Pten mutant and control hepatocytes using Lipofectamine 2000 Transfection Reagent (Invitrogen). Cells were harvested 48 hours after transfection.

\section{Determination of Serum Metabolic Indexes}

Serum was collected from 16-hour-fasted mice through cardiac puncture before sacrifice. Analysis for serum TG, cholesterol, non-esterified fatty acids, and 3-hydroxibutyrate were determined using kits provided by Wako chemical.

\section{Determination of Liver TG}

Liver lipid was extracted with chloroform/methanol (2/1) according to Folch method. The supernatant was used for TGs assay. The pellets were further lysed for DNA extraction. The TG content was determined using Triglyceride (GPO) Reagent Set (Thermo).

\section{Oil Red O Staining}

Frozen liver sections were briefly fixed in cold $10 \%$ formalin. Slides were stained in oil red O solution $(3 \mathrm{mg} / \mathrm{ml})$ and hematoxylin for counterstain.
Table 1. Real-Time Quantitative PCR Primers

\begin{tabular}{ll}
\hline \multicolumn{1}{c}{ Gene name } & \multicolumn{1}{c}{ Primer sequence } \\
\hline ACC1F & 5'-ACAGTGGAGCTAGAATTGGAC-3' \\
ACC1R & 5'-ACTTCCCGACCAAGGACTTTG-3' \\
FASF & 5'-AGCGGCCATTTCCATTGCCC-3' \\
FASR & 5'-CCATGCCCAGAGGGTGGTTG-3' \\
SREBP1F & 5'-AACGTCACTTCCAGCTAGAC-3' \\
SREBP1R & 5'-CCACTAAGTGCCTACAGAGC-3' \\
GlucokinaseF & 5'-CCCTGAGTGGCTTACAGTTC-3' \\
GlucokinaseR & 5'-ACGGATGTGGAGTGTTGAAGC-3' \\
SCD-1F & 5'-CCGGAGACCCTTAGATCGA-3' \\
SCD-1R & 5'-TAGCCTGTAAAAGATTTCTGCAAACC-3' \\
GAPDHF & 5'-GTCGGTGTGAACGGATTTGG-3' \\
GAPDHR & 5'-GACTCCACGACATACTCAGC-3' \\
\hline
\end{tabular}

\section{Glucose Tolerance Test}

The glucose tolerance test was performed on mice fasted for 16 hours at 1 and 3 months of age. ${ }^{4}$ Mice were given a single dose ( $2 \mathrm{~g} / \mathrm{kg}$ body weight) of D-dextrose (Sigma) by i.p. injection after a baseline glucose check. Circulating glucose levels were measured at indicated time after injection.

\section{Protein Electrophoresis}

Protein lysates $(75 \mu \mathrm{g})$ were loaded for each sample for electrophoresis. Membranes were probed with antibodies for total PTEN, AKT $(1+2+3)$, AKT2, p-AKT, acetyl-CoA carboxylase, p-FOXO1 (Ser 256), and p-aPKC (Thr410/403) from Cell Signaling, FAS (Upstate), and SREBP-1 Ab-1 (Clone 2A4) (Thermo). The membranes were also probed with vinculin and $\beta$-actin (Sigma) for loading control.

\section{Quantitative PCR}

Sequences used in generating $\mathrm{PPCR}$ are for acetyl-CoA carboxylase (ACC), FAS, SREBP-1c, Glucokinase (GK), and glyceraldehyde-3-phosphate dehydrogenase (primers listed in Table 1). Real-time PCR reactions were performed on Biorad iCycler. Relative fold changes were obtained by comparing the $\Delta \mathrm{Ct}$ between mutant and control groups.

\section{Statistical Analysis}

All data are subjected to multivariate analysis of variance. Fischer's LSD tests were used to determine differences among the groups. $P \leq 0.05$ is considered to be statistically significant. Data presented as mean \pm SEM.

\section{Results}

To investigate the function of AKT kinase in hepatic steatosis in Pten mutant mice, we crossed the PtenloxP/loxP: Alb-Cre ${ }^{+}$(Pten null) mice with the Akt2 ${ }^{-1-}$ mice (Akt2 null) to generate the double mutant Pten ${ }^{\text {loxP/loxP }}$; $A k 2^{-1-}$; $\mathrm{Alb}^{-\mathrm{Cre}^{+}}$(double mutant, DM). Figure 1A (left panel) shows the genotyping for Pten, Akt2 and Cre for each group of mice. Genotypes were confirmed with Western blot analysis (Figure 1A, right panel). Loss of Pten in- 
A
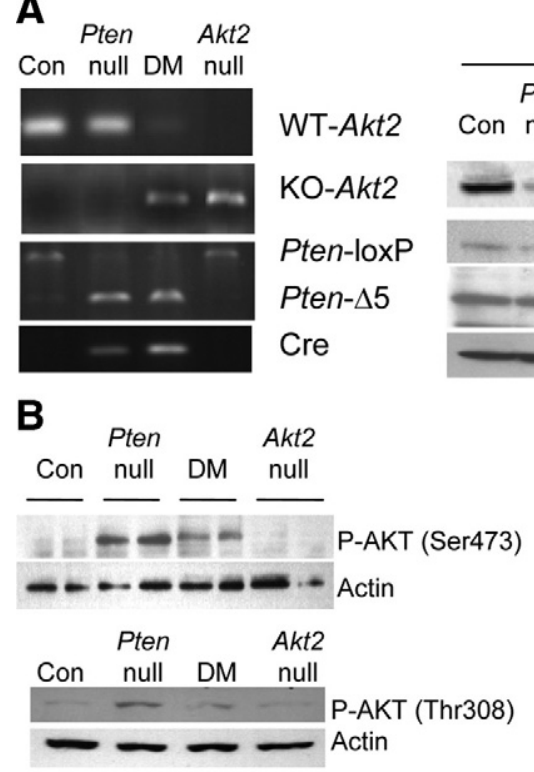
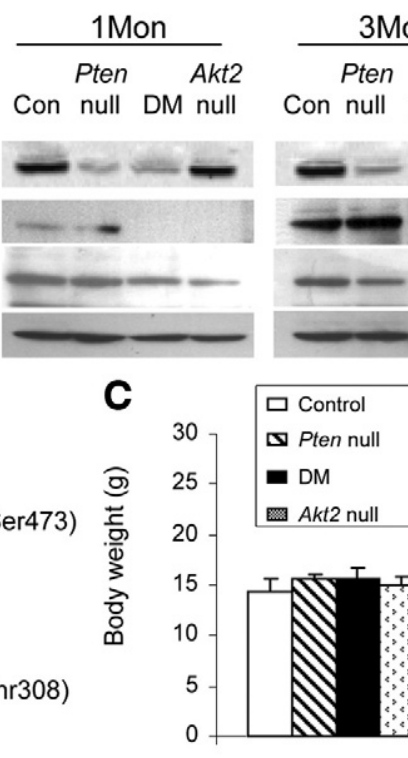

1 Mon
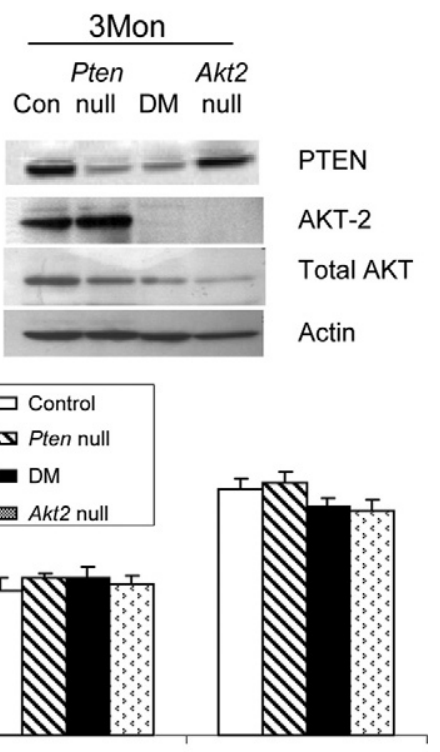

3 Mon
Figure 1. Generation of PTEN and AKT2 double mutant mice. A: Genotyping and Western blot confirmation of mice carrying various genotypes. Left: Representative PCR genotyping gel images of liver tissue lysates. Right: Representative Western blot images of lysates isolated from livers of various groups of mice. Blots were probed with PTEN (top) and AKT2 (middle). Actin was also blotted for loading controls (bottom). B: Representative Western blot analysis of phosphorylated AKTs Top panel, phospho-Ser 473; Bottom panel, phospho-Thr308. Phosphorylation on both residues was robustly induced by loss of PTEN (Pten null) compared with the control groups (Con). $\beta$-actin was detected as a loading control. C: Body weight was measured in mice carrying various genotypes. Open bar, control (Con); Striped bar Pten mutant only (Pten null); Solid bar, Pten and Akt2 double mutant (DM); patterned bar, Akt2 mutant only (Akt2 null). $n=3$ to 6 . duced robust phosphorylation on both Ser 473 and Thr 308 of AKT (Figure 1B). Phosphorylation of AKT was not visible when PTEN is intact in the control or AKT2 null mice. We observed that AKT (ser473) phosphorylation is decreased by approximately twofold in the DM group, as compared with the Pten null group (Figure 1B, top panel). Phosphorylation on Thr308 is more profoundly reduced to a level similar to controls when AKT2 is lost (Figure 1B, bottom panel). No compensatory increase of AKT1 was observed (data not shown). AKT3 level was not detectable in the liver lysates. The three mutant groups of mice are grossly morphologically normal with body weight comparable with the control mice, Pten ${ }^{\text {loxP/loxP }} ;$ Alb-Cre ${ }^{-}$ (Con) (Figure 1C).

\section{AKT2 Is Necessary for Lipid Accumulation in the Liver}

Loss of PTEN led to increased liver to body weight ratio (Figure 2A). Deletion of Akt2 in the DM group reduced liver weight, as compared with the Pten deletion mice. Loss of PTEN also led to accumulation of lipid in the hepatocytes. ${ }^{4}$ Loss of AKT2 led to a dramatic decrease in the severity of fatty liver (Figure 2B and Supplemental Figure 1 at $h$ ttp://ajp.amjpathol.org). Significantly less oil red $O$ staining is observed in livers of the DM mice compared with the Pten mutant. At 1 month of age, only Pten null mice accumulated lipid in the liver (see Supplemental Figure 1 at $h$ ttp://ajp.amjpathol.org). At 3 months of age, the Pten null mice displayed an even greater degree of steatosis, which was suppressed to almost control levels in the DM mice (Figure 2B). The lipid contents in the DM mice are dramatically reduced compared with the Pten null mice (Figure 2B). The control and the Akt2 ${ }^{-\prime-}$ mice (Akt2 null) did not stain with oil red $\mathrm{O}$, indicating little or no lipid deposition in the livers of these mice. Lipid accumulation occurs in both central vein (Figure 2B and
Figure 1sA) and portal triad area (Supplemental Figure 1B at http://ajp.amjpathol.org).

Loss of PTEN led to a two- and four fold increase of TG content in the liver of 1- and 3-month-old mice, respectively, as compared with the respective age matched controls (Figure 2C). Loss of AKT2 significantly blocks this effect of PTEN loss. The TG content in the DM mice is reduced to a level similar to the controls in 3-month-old mice, and even less than controls in the 1-month-old mice (Figure 2C). A reduction of approximately 2.5 -fold is observed in 3-month old DM mice vs. age matched Pten null mice. Deletion of Akt2 alone also led to >two fold decrease in liver TG, as compared with the control mice (Figure 2C). Thus, loss of AKT2 not only affected lipid accumulation induced by PTEN loss in the hepatocyte but also reduced lipid storage when not compounded with other factors that induces insulin signal.

\section{AKT2 Is Important for Lipogenesis in the Liver}

The accumulation of lipids in the liver of Pten mutant mice is associated with an increase in de novo lipogenesis. ${ }^{4}$ Serum index from the DM mice and Pten null groups suggests that there is no change in serum TG and nonesterified fatty acid levels (Table 2), two neutral lipids that can decrease if hepatic lipid export is inhibited. Cholesterol and 3-hydroxibutyrate levels were also not significantly different between the two groups, although AKT2 loss alone did lead to a significant reduction in 3-hydroxibutyrate (Table 2).

To investigate whether the PTEN loss-associated lipogenic effect is mediated by AKT2, we determined the levels of lipogenic enzymes ACC and FAS, enzymes that catalyze the two initial steps in de novo lipid synthesis. Both ACC and FAS mRNA levels are increased in the Pten null liver (significantly reduced $\Delta \mathrm{Ct}$ in the Pten null group), consistent with the severe fatty liver phenotypes 
A

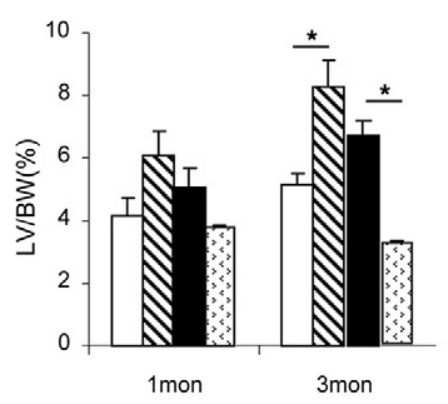

B

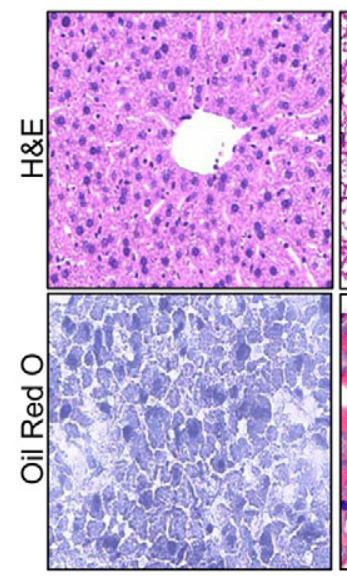

Pten null

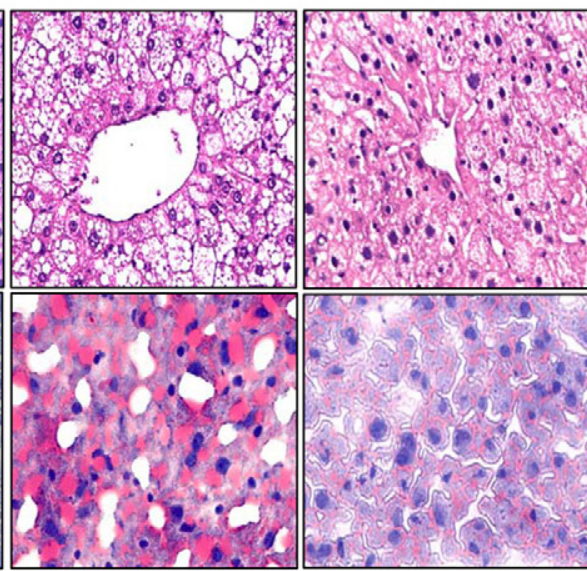

Double Mutant

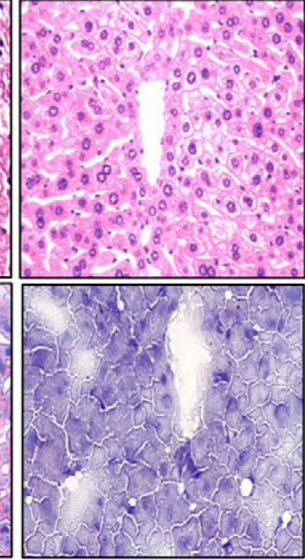

\section{C}

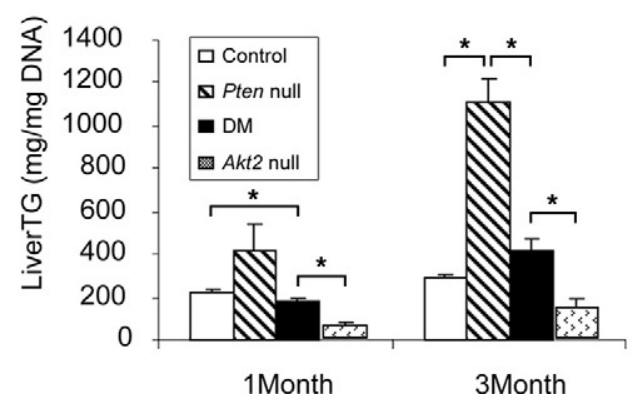

Figure 2. Loss of AKT2 reduces the lipogenic ability of liver. A: Loss of AKT2 (patterned bar) reduced the liver to body weight ratio, as compared with the control mice (open bar). The size of the liver is also reduced in the double mutant mice (solid bar) compared with Pten mutant only (stripped bar) mice. $n=8$. B: Liver tissues sections from 3-month-old mice carrying various genotypes. Left panel, control; Second from the left, Pten null only; Third from the left, Pten and Akt 2 double mutant (DM); Right panel, Akt 2 null only. Top panels, H\&E staining; Bottom panels, oil red O staining. Magnification $\times 20$. C: Quantification of liver triglyceride (TG) demonstrated loss of lipid accumulation in the Akt 2 null (patterned bar) and double mutant mice (solid bar), as compared with controls (open bar) and Pten mutant only (stripped bar). $n=3$ to $6 .{ }^{*} P \leq 0.05$ considered statistically significant difference. Data presented as mean \pm SEM. observed in these mice (Figure 3A, left two panels and Supplemental Figure 2 at http://ajp.amjpathol.org). Given the dramatic reduction in liver TG content in the DM mice, we expected that the DM mice would have the same level of ACC and FAS as control or even Akt null mice. Figure $3 A$ shows that expression of ACC is dramatically reduced (higher $\Delta \mathrm{Ct}$ ) in the DM group compared with the Pten null mice. The level of ACC expression in the DM mice is comparable with the controls or less. Loss of AKT2 also increased the $\Delta \mathrm{Ct}$ for amplifying $\mathrm{ACC}$ and calculated to be fivefold reduction in level of ACC compared with the controls (Figure 3A, left panel and Supplemental Figure 2 http://ajp.amjpathol.org). This lowered ACC is accounted for by decrease in both ACC1 and ACC2 expression (Table 3). Levels of FAS were reduced to a similar extent (Figure 3A, middle panels and Supplemental Figure 2A at http://ajp.amjpathol.org). AKT2 loss reduced FAS expression in the Pten null mice to the same level as the controls (Figure 3A, middle panels and Supplemental Figure 2A at http://ajp.amjpathol.org). These data confirmed that AKT2 is critical for the expression of liver lipogenic genes in the unstimulated (compare Akt2 null mice with controls) and stimulated (compare DM with Pten null) states. We also observed significant down-regulation of other genes involved in lipogenesis. Stearoyl-CoA desaturase 1, a key enzyme involved in lipogenesis is sensitive to changes in insulin signaling. We found that stearoyl-CoA desaturase 1 levels are dramatically induced as a result of PTEN loss (threefold) and are reduced back to the levels of control mice when AKT2 is concurrently lost (Table 3). AKT2 loss alone led to a >fivefold reduction in stearoyl-CoA desaturase 1. Together, our data supports a primary role of AKT2 in PTEN loss-induced lipogenesis.

We investigated the status of SREBP-1c, a critical transcriptional factor regulating lipogenesis in the liver and found that the expression of SREBP-1c is dependent on AKT2 (Figure 3A, right panels). When AKT2 is lost in the Akt2 null or DM mice, SREBP-1c expression is dramatically reduced. In the 1-month-old Akt2 null and DM mice, SREBP-1c levels were $50 \%$ and $25 \%$ of the controls, respectively (Figure 3A, right panels). When calculated to fold changes, loss of Pten dramatically induced SREBP-1c by fourfold, as compared with the controls, leading to a 16- and eightfold increase, comparing to the

Table 2. Serum Measurements of Pten and Akt Mutant Mice

\begin{tabular}{lrrrr}
\hline \multicolumn{1}{c}{ Measurements } & Con & Pten null & DM & Akt2 null \\
\hline Triglycerides $(\mathrm{mg} / \mathrm{dl})$ & $102.6 \pm 10.6$ & $87.4 \pm 7.9$ & $96.5 \pm 13.4$ & $94.1 \pm 5.4$ \\
Cholesterol $(\mathrm{mg} / \mathrm{dl})$ & $111.4 \pm 9.34$ & $100.0 \pm 13.3$ & $140.1 \pm 13.6$ & $144.7 \pm 5.3$ \\
NEFA $(\mathrm{mM})$ & $1.41 \pm 0.03$ & $1.22 \pm 0.13$ & $1.27 \pm 0.09$ & $1.22 \pm 0.14$ \\
3-HB $(\mathrm{mM})$ & $0.69 \pm 0.02$ & $0.84 \pm 0.07$ & $0.69 \pm 0.05$ & $0.40 \pm 0.03^{* * *}$ \\
\hline
\end{tabular}

Data reported as mean \pm SEM. $n=3$ to 6 , of 3-month-old mice.

***Different from all other groups. 

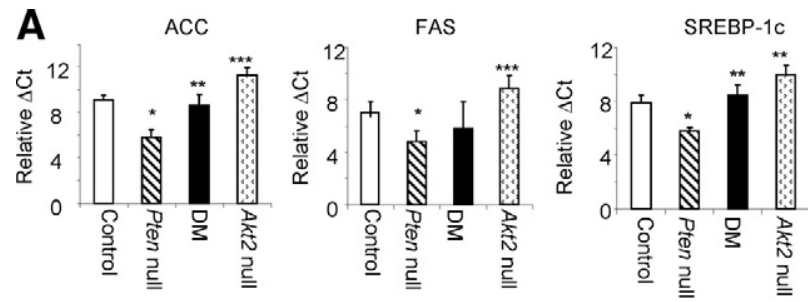

B
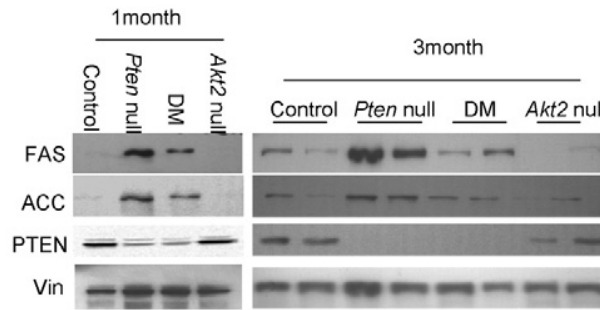

nSREBP-1C

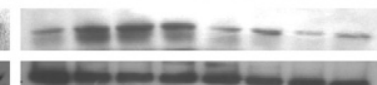

Actin $\longrightarrow-\infty$

Figure 3. Loss of AKT2 attenuates liver lipogenesis. A: qPCR analysis of key lipogenic enzymes and factors: acetyl CoA carboxylase (ACC), fatty acid synthase (FAS), and sterol regulatory element binding protein-1c (SREBP-1c). $n=3$. Relative $\Delta \mathrm{Ct}$ represents the $\mathrm{Ct}$ needed to reach threshold level subtracting the $\mathrm{Ct}$ needed for glyceraldehyde-3-phosphate dehydrogenase to reach the threshold as loading control. $P \leq 0.05$ is considered to be significant. *, different from

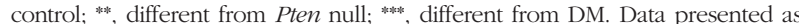
mean \pm SEM. B: Western analysis of ACC, FAS, and nuclear form of SREBP-1C levels in livers of various mouse models. Left, 1-month-old mice. Right, 3-month-old mice. Vincullin (Vin) and actin is detected as controls for loading.

Akt2 null and DM mice, respectively (Figure 3A, right panels). SREBP-1c levels in 3-month-old mice are consistently threefold lower in the two groups of mice lacking AKT2 than the controls (Supplemental Figure 2 at http://ajp.amjpathol.org). Loss of PTEN led to moderate but significant change in the expression of SREBP-1c (Supplemental Figure 2 at http://ajp.amjpathol.org). Loss of AKT2 alone or in DM mice resulted in an approximately four fold reduction in SREBP-1c and 2 expressions, as compared with their respective controls (Figure 3, Supplemental Figure 2 at $h t t p: / /$ ajp.amjpathol.org and Table 3). No changes were observed with PGC-1 in the Pten mutant mice (data not shown).

Similar to the gene expression increase, nuclear SREBP-1C, FAS, and ACC protein levels increased dramatically in the Pten null group (Figure 3B). This increase is blocked by loss of AKT2 in the DM mice. FAS protein levels in the DM group is similar to that of the controls,

Table 3. Loss of AKT2 Reduces the Expression of Lipogenic Genes

\begin{tabular}{lcccc}
\hline \multirow{2}{*}{$\begin{array}{c}\text { Gene of } \\
\text { interest }\end{array}$} & \multicolumn{4}{c}{ Genotype } \\
\cline { 2 - 5 } & Con & Pten null & DM & Akt2 null \\
\hline ACC1 & 1 & $1.11 \pm 0.11$ & $0.26 \pm 0.03^{* *}$ & $0.40 \pm 0.04^{* * *}$ \\
ACC2 & 1 & $1.04 \pm 0.17$ & $0.34 \pm 0.08^{* *}$ & $0.35 \pm 0.02^{* *}$ \\
SCD1 & 1 & $3.2 \pm 0.13^{\star}$ & $1.06 \pm 0.09^{* *}$ & $0.17 \pm 0.01^{* * *}$ \\
SREBP-2 & 1 & $0.90 \pm 0.16$ & $0.35 \pm 0.04^{* *}$ & $0.21 \pm 0.02^{* * *}$ \\
\hline
\end{tabular}

Data reported as mean \pm SEM with controls arbitrarily set as 1 . The relative expression of the gene of interest in other groups are compared to the control levels and reported as relative expression. $n=$ 3 of 3 -month-old mice.

${ }^{*}$ Different from control and Pten null groups.

${ }^{* *}$ Different from Pten null group.

***Different from all other groups. especially in the 3-month-old mice. Further reduction in FAS protein levels were observed in the Akt2 null mice, as compared with the DM and the control groups, indicating that other factors than AKT2 may also play roles in the protein levels of FAS in these mice.

\section{Loss of AKT2 Abrogates the Effect of Pten Deletion on Glucose Tolerance}

The hepatocyte-specific, PTEN-deficient mice displayed improved glucose tolerance. ${ }^{4}$ This was completely reversed by concomitant removal of Akt2 such that the glucose excursion was indistinguishable from $A k t 2^{-1-}$ mice. Both $A k t 2^{-1-}$ and DM mice displayed hyperglyce-
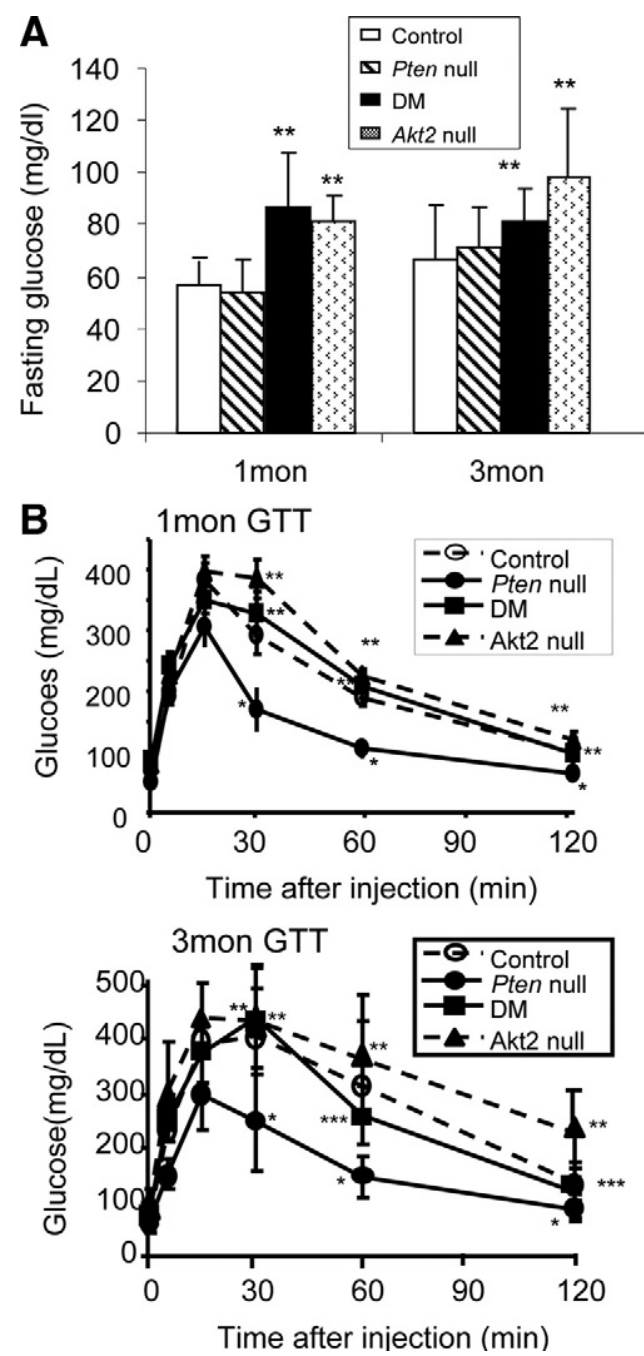

Figure 4. AKT2 loss reduces glucose tolerance of hepatocyte Pten mutant mice. A: Plasma glucose levels were determined in mice fasted overnight. $n=8$. Open bar, control (Con); Stripped bar, Pten mutant (PTEN null); Solid bar, double mutant (DM), Patterned bar, Akt2 mutant (AKT2 null). Data presented as mean \pm SEM. B: Glucose tolerant test (GTT) shows intolerance to clear glucose load in Akt2 null (dashed line with triangle) or DM (solid lie with square), as compared with the hepatocyte Pten null (solid line circle) mice. Controls, dashed line with open circle. Top panel, 1-month-old mice. Bottom panel, 3-month-

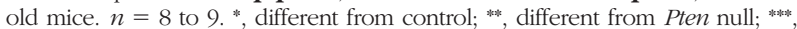
different from double mutant (DM). Data presented as mean \pm SEM. $P \leq 0.05$ considered statistically significant difference. Con, open bar; Pten null, stripped bar; double mutant (DM) mice, solid bar; and Akt2 null, patterned bar. Data presented as mean \pm SEM. $n=3$ to 6 . 


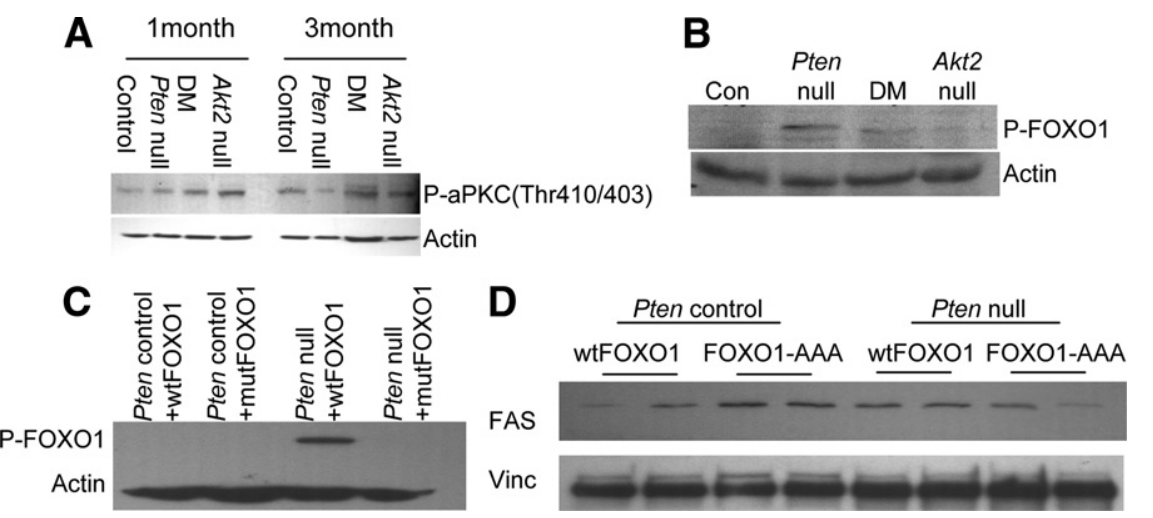

\begin{abstract}
Figure 5. AKT2 not atypical PKC activity is associated with the reduced lipid accumulation in the liver of double mutant mice. A: Representative Western blot analysis of phosphorylated atypical PKC. B: Representative Western blot analysis of phosphorylated FOXO1. C: PhosphoFOXO levels of Pten control and mutant cells transfected with wild-type (wt) FOXO or nuclear localized mutant FOXO (FOXO-AAA). Actin is used as loading control. D: FAS levels in cells transfected with the indicated FOXO constructs. Vinculin (Vin) is used as loading control.
\end{abstract}

mia and glucose intolerance, as compared with Pten mutant groups (Figure 4). Loss of AKT2 led to an approximately 25 to $30 \%$ increase in fasting glucose levels in the $\mathrm{DM}$ and Akt2 null groups, as compared with the control and Pten null groups at 1 month of age (Figure 4A). This trend continued at 3 months of age. Indeed, the glucose tolerance test curve of the DM is at the same level as the control mice whereas PTEN loss induced a downshift of the curve (Figure 4B, bottom panel). The glucose tolerance test curve indicates that the Akt2 mutant mice are deficient in clearing glucose as previously reported. ${ }^{7}$ No significant differences were observed with fasting insulin levels among any of the groups (data not shown).

\section{The Reduction of Lipogenesis and Glucose Sensitivity Is Associated with AKT, not PKC, Activation}

Signals downstream of PI3K may diverge in their functions during insulin signal transduction. Atypical PKC (aPKC) expression has been shown to induce the expression of lipogenic genes in livers lacking PI3K enzymes. ${ }^{2}$ We found that the levels of phospho-aPKC are not decreased in the DM group, as compared with the Pten null group (Figure 5A). If any change is seen, there are moderate increases in phospho-aPKC in the two groups lacking AKT2, a possible consequence of feedback activation in PI3K signaling upstream of AKT due to lack of AKT2.

Increasing evidence has shown that the forkhead transcriptional factor FOXO1 may play a role in the regulation of lipogenesis. ${ }^{9,10}$ Phosphorylation of FOXO1 by AKT leads to its nuclear exclusion and inability to assume its transcriptional activity. When PTEN is lost, the level of phosphorylated FOXO1 becomes detectable (Figure 5B). Its level is reduced in DM groups and further reduced when AKT2 is lost by itself (Figure 5B). These data indicate that $\mathrm{FOXO1}$ phosphorylation by AKT2 may mediate the effect of AKT2-regulated lipogenesis.

To evaluate whether phosphorylation of FOXO led to the increased lipogenesis effect seen with Pten mutation, we introduced wild-type FOXO (wtFOXO) and a nonphopshorylatable mutant of FOXO (FOXO-AAA) to the hepatocyte cell lines that we established from the control and mutant mice. ${ }^{8}$ When introduced to the control mice, neither wtFOXO nor mutant FOXO-AAA are phosphory- lated, indicated by the lack of phospho-FOXO in the transfected Pten control cell lines (Figure 5C). When introduced to the Pten mutant cell line, the wtFOXO is readily phosphorylated (Figure $5 \mathrm{C}$ ), consistent with the phosphorylation status of AKT in the cells. The FOXO-AAA mutant, however, is not phosphorylated, as indicated by the lack of phospho-FOXO band in this sample group (Figure $5 \mathrm{C}$ ). We investigated the effects of introducing FOXO-AAA mutant to the Pten mutant cells and compared it to the cells that received wtFOXO. The FOXO-AAA mutant transfected cells showed reduced levels of FAS compared with the wt-FOXO transfected cells (Figure 5D). Interestingly, the FOXO-AAA induced FAS protein levels in the Pten control cells. The reason for this is not clear and may not be dependent on the signals from PI3K/AKT as this signal axes are not active, as indicated by the lack of FOXO phosphorylation in the wtFOXO-transfected control cells.

\section{Discussion}

PTEN is a lipid phosphatase that negatively regulates insulin signaling. Loss of PTEN leads to lipid accumulation in hepatocytes and steatosis in the liver. How PTEN loss results in this metabolic phenotype is not clear. Our present study using a combination of Pten and Akt2 deletion specifically evaluated the function of AKT2 in liver metabolism. Loss of AKT2 alone or in combination with AKT3 renders the mice glucose and insulin intolerant. ${ }^{7,11,12}$ Studies using cells isolated from the AKT2 mice and cultured cell lines have shown that AKT2 is required for insulin-dependent uptake of glucose in adipocytes. 6,13,14 Our results show that germline loss of AKT2 can efficiently block both the lipid accumulation and the improved glucose tolerance induced by hepatocyte-specific Pten deletion. AKT2 loss alone also reduced lipogenesis in addition to rendering the mice mildly glucose intolerant as reported previously. ${ }^{7}$ Loss of PTEN mimics the constitutive engagement of the insulin receptor and accumulation of $\mathrm{PIP}_{3}$ in the cells. Under this condition, the increased lipogenesis and expression of lipogenic genes, such as FAS, SREBP-1c, and ACC, depend on AKT2 to be present. However, our study does not exclude the involvement of other factors in hepatic lipogenesis, especially given the fact that slightly more reduction of lipogenic expression is observed with the 
Akt2 $^{-1-}$ mice than in DM mice. The effect of AKT2 loss, however, is much more significant, and thus, is likely an essential regulator for the Pten loss induced lipid accumulation in hepatocytes. Our observation supports a pivotal role for AKT2 in lipogenesis.

These observations appear to be in disagreement with analysis in mice lacking two isoforms of $\mathrm{PI}_{3} \mathrm{~K}^{2}{ }^{2}$ In the PI3K-deficient mice, the reduction of FAS and SREBP-1C mRNA expression was rescued by adenovirus expression of ca-PKC $\lambda$ but not myr-AKT. Several explanations may account for these disagreements. First, the use of a nonspecified isoform of myr-AKT in the PI3K-deficient mice study makes it difficult to directly compare our data with the atypical PKC data. How AKT2 and AKT1 differ functionally on the molecular level is not clear. If AKT1 is the isoform used for myristylation, it may not have similar effect on srebp-1c and FAS expression as AKT2 activation. Secondly, direct targeting of AKT to the membrane with the attachment of myristylation signal may not mimic the endogenous activation of AKT. Finally, the PI3K-deficient mice lack the function of lipogenesis, whereas PTEN-deficient mice are functionally enhanced in lipogenesis. ${ }^{2,4}$ The differential effects seen with the two studies may be a difference between stimulated and basal levels of lipid homeostasis regulation. Loss of $\mathrm{PKC}^{15}$ or AKT2 by themselves both led to reduced lipid accumulation in the liver, suggesting that both may maintain the basal lipid homeostasis. Our study showed that AKT2 is also critical for liver lipid accumulation in the stimulated stage of PTEN loss. Without AKT2, the expression of lipogenic genes such as ACC, FAS, and the lipogenic transcriptional factor SREBP-1c cannot be induced by activation of PI3K signaling through PTEN loss. While expression of $P K C \lambda$ is sufficient to drive the expression of these genes, the requirement of PKC $\lambda$ in the stimulated stage remains to be tested. In the stimulated stage mimicked by loss of PTEN, lipogenesis in hepatocytes is partially reversed by expression of a nuclear localized constitutively active FOXO. This transcriptional factor has been shown to play a role in lipogenesis previously. ${ }^{9,10}$ Our data support the idea that FOXO may be partially responsible for lipogenesis occurring in hepatocytes when $\mathrm{PIP}_{3}$ levels are accumulated. In conditions where AKT is not activated (as in the case of control cells), inhibition of FOXO activity may not have a significant effect.

In summary, we have explored the relationship between AKT2 and PTEN regulated metabolic changes in the liver. Our data strongly support the indispensable function of AKT2 in liver lipid accumulation and regulation of glucose tolerance. While this manuscript was in the final stage of review, Leavens et al reported that AKT2 is also necessary for hepatic lipid accumulation in insulin resistance models. ${ }^{16}$ We further observed that this change in metabolic signal led to a 3-month delay in tumor development (data not shown). How this branch of the signaling downstream of PTEN may contribute to the development of liver cancer in this mode ${ }^{3,8}$ remains to be investigated.

\section{Acknowledgments}

We thank Dr. Neil Kaplowitz, Dr. Sarah Hamm-Alvarez, and Dr. Deborah Johnson for their wonderful suggestions and assistance in proofreading this manuscript.

\section{References}

1. Myers MP, Stolarov JP, Eng C, Li J, Wang SI, Wigler MH, Parsons R, Tonks NK: P-TEN, the tumor suppressor from human chromosome 10q23, is a dual-specificity phosphatase. Proc Natl Acad Sci USA 1997, 94:9052-9057

2. Taniguchi CM, Kondo T, Sajan M, Luo J, Bronson R, Asano T, Farese $R$, Cantley LC, Kahn CR: Divergent regulation of hepatic glucose and lipid metabolism by phosphoinositide 3-kinase via Akt and PKClambda/zeta. Cell Metab 2006, 3:343-353

3. Horie Y, Suzuki A, Kataoka E, Sasaki T, Hamada K, Sasaki J, Mizuno K, Hasegawa G, Kishimoto $H$, lizuka M, Naito M, Enomoto K, Watanabe S, Mak TW, Nakano T: Hepatocyte-specific Pten deficiency results in steatohepatitis and hepatocellular carcinomas. J Clinl Invest 2004, 113:1774-1783

4. Stiles B, Wang Y, Stahl A, Bassilian S, Lee WP, Kim YJ, Sherwin R, Devaskar S, Lesche R, Magnuson MA, Wu H: Liver-specific deletion of negative regulator Pten results in fatty liver and insulin hypersensitivity [corrected]. [erratum appears in Proc Natl Acad Sci USA: 2004 Apr 6;101(14):5180]. Proc Natl Acad Sci USA 2004, 101:2082-2087

5. Whiteman EL, Cho H, Birnbaum MJ: Role of Akt/protein kinase B in metabolism. Trends Endocrinol Metab 2002, 13:444-451

6. Bae SS, Cho H, Mu J, Birnbaum MJ: Isoform-specific regulation of insulin-dependent glucose uptake by Akt/protein kinase B. J Biol Chem 2003, 278:49530-49536

7. Cho H, Mu J, Kim JK, Thorvaldsen JL, Chu Q, Crenshaw EB 3rd, Kaestner KH, Bartolomei MS, Shulman GI, Birnbaum MJ: Insulin resistance and a diabetes mellitus-like syndrome in mice lacking the protein kinase Akt2 (PKB beta). Science 2001, 292:1728-1731

8. Xu X, Kobayashi S, Qiao W, Li C, Xiao C, Radaeva S, Stiles B, Wang $\mathrm{RH}$, Ohara N, Yoshino T, LeRoith D, Torbenson MS, Gores GJ, Wu H, Gao B, Deng CX: Induction of intrahepatic cholangiocellular carcinoma by liver-specific disruption of Smad4 and Pten in mice. J Clin Invest 2006, 116:1843-1852

9. Valenti L, Rametta R, Dongiovanni P, Maggioni M, Fracanzani AL, Zappa M, Lattuada E, Roviaro G, Fargion S: Increased expression and activity of the transcription factor FOXO1 in nonalcoholic steatohepatitis. Diabetes 2008, 57:1355-1362

10. Zhang W, Patil S, Chauhan B, Guo S, Powell DR, Le J, Klotsas A, Matika R, Xiao X, Franks R, Heidenreich KA, Sajan MP, Farese RV, Stolz DB, Tso P, Koo SH, Montminy M, Unterman TG: FoxO1 regulates multiple metabolic pathways in the liver: effects on gluconeogenic, glycolytic, and lipogenic gene expression. J Biol Chem 2006, 281:10105-10117

11. Garofalo RS, Orena SJ, Rafidi K, Torchia AJ, Stock JL, Hildebrandt AL, Coskran T, Black SC, Brees DJ, Wicks JR, McNeish JD, Coleman KG: Severe diabetes, age-dependent loss of adipose tissue, and mild growth deficiency in mice lacking Akt2/PKB beta. J Clin Invest 2003, 112:197-208

12. Dummler B, Tschopp O, Hynx D, Yang ZZ, Dirnhofer S, Hemmings BA: Life with a single isoform of Akt: mice lacking Akt2 and Akt3 are viable but display impaired glucose homeostasis and growth deficiencies. Mol Cell Biol 2006, 26:8042-8051

13. $\mathrm{Ng} \mathrm{Y,} \mathrm{Ramm} \mathrm{G,} \mathrm{Lopez} \mathrm{JA,} \mathrm{James} \mathrm{DE:} \mathrm{Rapid} \mathrm{activation} \mathrm{of} \mathrm{Akt2} \mathrm{is}$ sufficient to stimulate GLUT4 translocation in 3T3-L1 adipocytes. Cell Metabolism 2008, 7:348-356

14. Yun SJ, Kim EK, Tucker DF, Kim CD, Birnbaum MJ, Bae SS: Isoformspecific regulation of adipocyte differentiation by Akt/protein kinase Balpha. Biochem Biophys Res Commun 2008, 371:138-143

15. Matsumoto M, Ogawa W, Akimoto K, Inoue H, Miyake K, Furukawa K, Hayashi $Y$, Iguchi H, Matsuki Y, Hiramatsu R, Shimano H, Yamada N, Ohno S, Kasuga M, Noda T: PKClambda in liver mediates insulininduced SREBP-1c expression and determines both hepatic lipid content and overall insulin sensitivity. J Clin Invest 2003, 112:935-944

16. Leavens KF, Easton RM, Shulman GI, Previs SF, Birnbaum MJ: AKT2 is required for hepatic lipid accumulation in models of insulin resistance. Cell Metabolism 2009, 10:405-418. 